\title{
On the broad tails in breaking time distributions of vibrated clogging arches
}

\author{
Angel Garcimartín ${ }^{1, *}$, Bruno V. Guerrero ${ }^{1}$, Alexandre Nicolas ${ }^{2}$, Rodrigo C. Barbosa da Silva ${ }^{1}$, and Iker Zuriguel ${ }^{1}$ \\ ${ }^{1}$ Depto. de Física y Matemática Aplicada, Facultad de Ciencias, Universidad de Navarra, 31080 Pamplona, Spain. \\ ${ }^{2}$ Institut Lumière Matière (CNRS \& Univ. Lyon 1), 10 rue Ada Byron, 69622 Villeurbanne Cedex, France
}

\begin{abstract}
Flowing grains can clog an orifice by developing arches, an undesirable event in many cases. Several strategies have been put forward to avoid this. One of them is to vibrate the system in order to undo the clogging. Nevertheless, the time taken to break an arch under a constant vibration has a distribution displaying a heavy tail. This can lead to a situation where the average breaking time is not well defined. Moreover, it has been observed in some experiments that these tails tend to flatten for very long times, exacerbating the problem. Here we will review two conceptual frameworks that have been proposed to understand the phenomenon and discuss their physical implications.
\end{abstract}

\section{Introduction}

Clogging is a distinct feature of grains flowing through a constriction or a duct. An arch (a mechanically stable structure) can be spontaneously formed and halt the flow. In order to break this arch and restore the flow $(i . e$. unclog the granular medium) an external perturbation is required. This is often done in the form of a vibration. The endurance of arches against the external vibration can be quantified by means of the time that it takes to break them from the moment when the vibration is applied; this time lapse is called $t_{b}$. A feature that has been found in many different systems is that the distribution of breaking times, $P\left(t_{b}\right)$, displays a heavy tail [1-3], which often flattens at very long times .

In practice, survival analysis is often used to describe the results. The survival function, also known as complementary cumulative probability distribution, is defined as

$$
S(t) \equiv P\left(t_{b}>t\right) \equiv \int_{t}^{\infty} P\left(t^{\prime}\right) d t^{\prime}
$$

In other words, $S(t)$ is the probability that an arch is still unshattered after a time $t$. If $P\left(t_{b}\right) \sim t_{b}^{-\alpha}$ then $S\left(t_{b}\right) \sim$ $t_{b}^{-\alpha+1}$. To be physically realistic, the power law is only valid for a range of times. The power law must break down at short times (otherwise, it would diverge for $t \rightarrow 0$ ) and after a long time the vibration can further alter the arch stability by making it more resistant (therefore, flattening the survival function).

We have carried out several sets of experiments, some of which have been previously reported [3-5]. The ones used here consist of a monolayer of grains enclosed between two vertical glass plates. An orifice at the base lets the grains flow until an arch is formed, halting the outpouring. Then, an external energy input is supplied to

*e-mail: angel@unav.es

A video is available at https://doi.org/10.48448/70fv-hz89

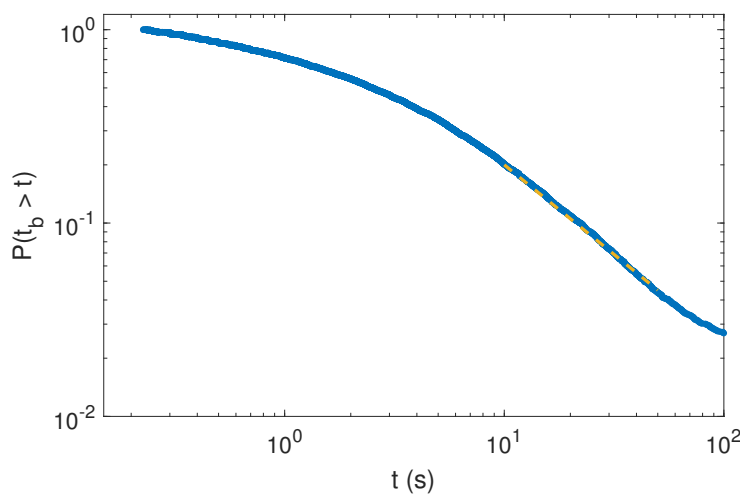

Figure 1. The blue points are the experimental survival function of more than 6000 arches (they look like a continuous trait). They were obtained for a two dimensional silo with a vibrated base; the exit orifice is $2.3 \mathrm{~mm}$ wide (beads have a diameter of $1 \mathrm{~mm}$ ). The sinusoidal vibration has a frequency of $f=90 \mathrm{~Hz}$ and an acceleration amplitude of $0.025 \mathrm{~g}$. The dashed line is a linear fit between $t=10 \mathrm{~s}$ and $t=50 \mathrm{~s}$, giving $\alpha=-1.93$.

break the arch and restore the flow. The time needed to break the arch, $t_{b}$, is recorded. These experiments have been performed with several protocols. Sometimes, the whole silo is vibrated; in other cases, only the base is shaken. The vibration may be an acceleration ramp, or a perturbation of constant amplitude. Irrespective of the specific procedures, a heavy tail for large $t_{b}$ is a feature that often appears in these experiments. A typical survival function of $t_{b}$ obtained from a particular experiment (see caption for details) is shown in Fig. 1.

Such distributions imply that a characteristic time scale is lacking. Apart from fundamental questions about the phenomenon itself, this leads to a disturbing possibility: it may be impossible to predict whether or not 
a given vibration will be able to restore the flow in a finite time. This stems from the fact that the integral $\left\langle t_{b}\right\rangle=\int t_{b} P\left(t_{b}\right) d t_{b}$ may not converge, as $P\left(t_{b}\right) \sim t_{b}^{-\alpha}$. Thus, if $\alpha \leq 2$ the integral diverges as the experimental time grows: the mean is dominated by large events. In other words, arches may outlast any finite time threshold that one can set, and therefore unclogging with gentle vibrations may be unfeasible in this circumstance. This represents a problem whenever a realistic, finite time is considered. The fact that the distribution flattens for very long times only worsens the problem. In fact, there may exist unbreakable arches that cannot be destroyed with a constant amplitude vibration even if it is applied for very long periods.

The granular flow through a bottleneck is a paradigm that has been used as a model to explain the features of other systems, such as macroscopic active matter. It has been proposed that the clogging transition can be described with three universal variables, which can be traced to the loads and densities of granular media. Heavy tails appear in many of those systems $[1,6,7]$ with features similar to those found in granular matter. Therefore, this study can have broad resonance in other fields.

In the following, we will review two explanations that can be given to describe and better understand the appearance of heavy tails in the distribution of $t_{b}$ when arches are submitted to a vibration. Remark that these two explanations are not mutually exclusive.

\section{Trap model: energy barrier distribution}

The conceptual framework of this model is to consider the stable arch as a point located inside a potential well, which describes the potential energy associated to a stable configuration and the small deviations from it. The form of this well is determined by the arch geometry, and in general, it will be difficult to find explicitly, as we do not know how to relate the arch shape to its resistance by means of an analytical expression. The different clogging arches will therefore define an energy landscape. A vibration applied to the arch can be likened to a thermal agitation, because experiments indicate that the evolution of the potential (linked to the creeping motion of the arch beads) is much slower than the vibration frequency. Thus, the point representing the arch shape can move inside the well and eventually escape from it, meaning that the arch breaks down. Taking into account the frictional forces, this process can be described by a Langevin equation:

$$
\ddot{r}=-\frac{\partial V}{\partial r}+f+\xi(t)
$$

where $r$ is the position of a variable representing the arch shape, $V$ stands for the potential, $f$ for the frictional forces, and the vibration is represented by $\xi(t)$ (a 'noise'). This concept is summarized in Fig. 2.

The escape rate from a potential well was worked out by Kramers [8]. Here, we have an energy landscape with different well depths (Fig. 2, b). In fact, the distribution of energy barriers $E_{b}$ can be inferred from experimental

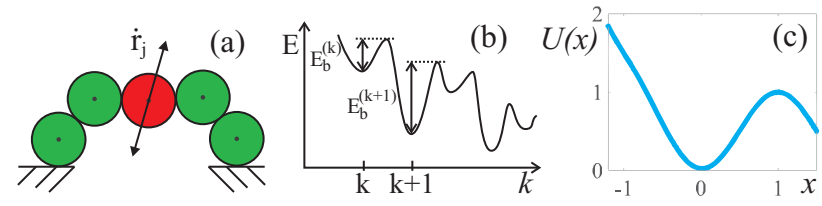

Figure 2. Trap model for the energy landscape of arch configurations. (a) Beads in the arch explore different configurations by minute displacements; (b) Each stable configuration can be thought of as a point at the bottom of a potential well; each well $k$ corresponds to one arch, and the whole set of arches describe an energy landscape (c) A generic energy barrier used in the numerical simulations. Figure adapted from [9].

results: in [9], it was derived from the experimental data on the perturbation amplitude needed to break the arches. Under some reasonable assumptions, it was found that $E_{b}$ follows a Weibull distribution

$$
p\left(E_{b}\right)=\frac{1}{a E_{b}^{\star}}\left(\frac{E_{b}}{E_{b}^{\star}}\right)^{\frac{1-a}{a}} e^{-\left(E_{b} / E_{b}^{\star}\right)^{1 / a}}
$$

where $E_{b}^{\star}$ is an energy scale of the system, and $a$ is a shape factor of the distribution. The experimental results imply that $a=2$, as argued in [9].

The distribution of escape times $P\left(t_{b}\right)$ cannot be calculated analytically for all cases, but a reasonable approximation yields

$$
P\left(t_{b}>t\right) \approx e^{-(\epsilon \ln t)^{1 / a}}
$$

where $\epsilon$ depends on the friction coefficient, the vibration intensity and $E_{b}^{\star}$.

Numerical solutions with an energy well shape as that in Fig. 2, c provide a result that closely resembles the survival function found in experiments (Fig. 1), meaning, a heavy tail which tend to flatten for long times.

In summary, this model can reproduce experimental results with a sensible distribution of energy barriers. Each arch is inside a well that defines the stable configurations. Exploration of this potential well, and eventually escaping from it (entailing breakage) is enabled by the external vibration. The escape times can be computed numerically for a given distribution of energy barriers, and they are consistent with heavy tails that flatten for very long times, as observed in experiments.

\section{Ageing}

An alternative explanation to justify the origin of the broad tails in the distribution of breaking times observed in the experiments is to model the temporal evolution of the arch properties as a random walker. The position of the random walker is given by a variable related to the arch geometry, so a change in the arch shape is equivalent to a step of the random walker. In general, the length of the steps and the times at which these steps are taken are not fixed. The path takes place in a space where the boundary is the frontier beyond which no stable configuration is to be found, meaning, the point where arches break down. It is of course 
an absorbing boundary, because the random walker cannot come back once it reaches it. Breaking times can be inerpreted as a first passage time through that boundary. The energy needed by the arch to explore this space ( $i$. $e$. the energy needed to deform it plastically) is provided by the external perturbation. Depending on the specific features of the random walk, this process can be history dependent.

This explanation was elaborated to better understand the dynamics of arches while they were being vibrated. In a series of experiments $[4,5]$ and numerical simulations [10] the positions of the beads in the arch where registered along time, with a high sampling frequency. In order to define the arch geometry, the angles between two consecutive beads were used, taken as a vector; or, alternatively, the standard deviation $\sigma$ of that set of angles was used. The latter misses some details, but nevertheless it captures in a single number the more salient episodes of the dynamics, which is composed of jerking motions interspersed with almost quiescent time periods (see [4]). It was therefore used as a proxy to describe the arch irregularity.

With these experimental results at hand, it is possible to analyze the flattening of the survival function (see in Fig. 1 how the curve changes the decreasing trend for $t_{b}>$ $50 \mathrm{~s}$ ). This means that once a certain time is reached, the risk (as characterized by the hazard function, which is related to the derivative of the survival function) is lower.

Ageing is a term often used in the context of polymers and other soft matter [11]. In the glass transition and in some other cases, a dependence on the logarithm of time is observed, and has been successfully explained with the help of some models. In those systems, a rapid quench from a high temperature to a temperature below the glass transition point leads to a dynamic change of the system properties: they depend on the time after the quench, and the change rate gradually slows down. This can be demonstrated by waiting a time $t_{w}$ after the quench and then measuring a given property along time: the rate of change will be slower as $t_{w}$ increases.

In order to check whether such a phenomenon is present in the experiment, we can calculate the time autocorrelation function of $\sigma$ at different waiting times. As this variable captures to some extent the evolution of the arch geometry, it will slowly decrease with time. Remark that the position of the beads will nevertheless be strongly correlated, so the decrease of the autocorrelation will be small. The key aspect is whether the correlation function changes with $t_{w}$. This is shown in Fig. 3, where the twotime autocorrelation function is plotted. As can be seen, $C\left(t_{w}, t\right)$ decreases along time. The longer $t_{w}$, the smaller the decrease of $C$. After about 10 seconds, the changes in the correlation function with $t_{w}$ are too small to be noticed.

A simple framework in which this phenomenon fits nicely is a model called continuous-time random walk (CTRW). It considers a random walker, as in Brownian motion, but irregular steps are taken at irregular time intervals. In CTRW models, these steps are sampled from a continuous distribution of times. The picture therefore would be that of a configuration space that is randomly explored by the arch shape (as embodied by a variable such as $\sigma$ ). Rearrangements of the beads would correspond to

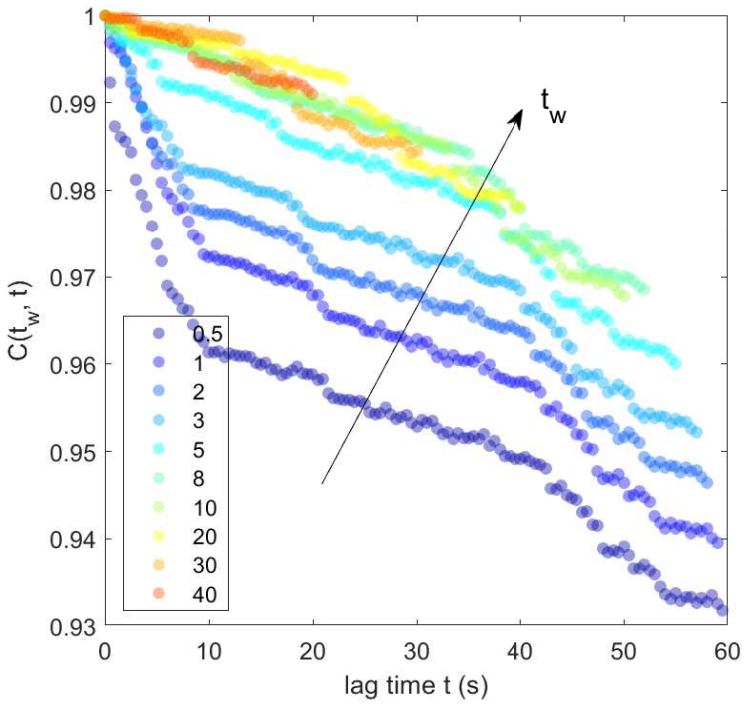

Figure 3. Time correlation function of $\sigma$ for different waiting times, from 0.5 to 40 seconds, as indicated in the legend. Figure adapted from [4].

the jumps of the random walker, and interludes to quiescent times of the CTRW.

This model has been studied by Merrigan and coworkers [10], who tuned a CTRW to reproduce the results of numerical simulations. This boils down to the choice of a suitable distribution for the times at which the walker jumps. They included a broad distribution of quiescent times (in fact, a stretched exponential distribution similar to the proposed for the aforementioned trap model). Later, Guerrero and coworkers [5] estimated the quiescent times distribution from experimental data and they found that this CTRW reproduces many of the features of the experiments, although quantitative agreement is sometimes lacking. In particular, absence of ergodicity -as revealed by the difference between ensemble averaged and time averaged mean squared displacements of the variable describing the geometry- was found both in the experiments and the model.

\section{Discussion}

The distribution of the breaking times of vibrated arches often display broad tails, which may even tend to flatten for very long times. This means that the phenomenon lacks a characteristic time scale. Heavy tails are a disturbing issue as they can lead to diverging averages for the breaking times, which in turn implies that vibration may not succeed in breaking arches and restoring the flow.

To date, two models have been put forward to rationalize these features. The first of them, the trap model, considers a particular distribution of potential wells, each well corresponding to a single arch configuration. It does not include any dynamics except the escape rate from the well; escaping from the well means that the arch breaks down. The distribution of breaking times stem from the 
energy barrier distribution, which is in turn related to the wide range of arches -each one with a given robustnessable to clog the orifice. The second model, a continuous time random walk, uses a specific time distribution for the intervals between consecutive steps directly related with the breaking times distribution. The dynamics of the random walker are here included as an ingredient of the model. In this model, memory effects are at play, as it is a history dependent process.

Note that both models are not incompatible, as the two mechanisms can be acting at the same time. It would be interesting to extend and couple both models, so that a random walker explores an energy landscape with different potential wells corresponding to an assortment of shapes. An arch could therefore escape from a well, fall into another one, and drift around this landscape until it either reaches the boundary or falls in a well so deep that the vibration cannot provide enough energy to extract it from there.

\section{Acknowledgements}

A. G. and I. Z. acknowledge funding from Ministerio de Economía y Competitividad (Spanish Government) through projects FIS2014-57325 and FIS2017-84631, MINECO/AEI/FEDER, UE. A. N. is funded by CNRS (Centre Nationale de la Recherche Scientifique, France). B. G. and R. BdS. thank Asociación de Amigos de la Universidad de Navarra for support through a scholarship. We thank L. I. Pagonabarraga and A. Pugnaloni for fruitful discussions and L. F. Urrea for technical help.

\section{References}

[1] I. Zuriguel et al., Sci. Rep. 4, 07324 (2014)

[2] C. Lozano, Ph. D. thesis: Estabilidad de los arcos en un medio granular frente a vibraciones (2014), available at https://www.unav.edu/ web/laboratorio-medios-granulares/publicaciones/ tesis-doctorales

[3] C. Lozano, I. Zuriguel, A. Garcimartín, Phys. Rev. E 91, 062203 (2015)

[4] B. V. Guerrero, L. A. Pugnaloni, C. Lozano, I. Zuriguel, A. Garcimartín, Phys. Rev. E 97, 042904 (2018)

[5] B. V. Guerrero, B. Chakraborty, I. Zuriguel, A. Garcimartín, Phys. Rev. E 100, 032901 (2019)

[6] K. Harth, J. Wang, T. Börzsönyi, R. Stannarius, Soft Matter, 16, 8013-8023 (2020)

[7] B. Blanc, J.-C. Géminard, L. A. Pugnaloni, Eur. Phys. J. E 37, 112 (2014)

[8] H. Kramers, Physica 7, 284 (1940)

[9] A. Nicolas, A. Garcimartín, I. Zuriguel, Phys. Rev. Lett. 120, 198002 (2018)

[10] C. Merrigan, S. K. Birwa, S. Tewari, B. Chakrabory, Phys. Rev. E 97, 040901(R) (2018)

[11] P. Papon, J. Leblond, P. H. E. Meijer, Physique des transitions de phases (Dunod, Paris, 1999). 